\title{
Nanoparticle-mediated drug delivery to treat infections in the female reproductive tract: evaluation of experimental systems and the potential for mathematical modeling
}

This article was published in the following Dove Press journal:

International Journal of Nanomedicine

\author{
Lee B Sims' \\ Hermann B Frieboes ${ }^{1-3}$ \\ Jill M Steinbach-Rankins ${ }^{1,3-5}$ \\ 'Department of Bioengineering, \\ University of Louisville, Louisville, KY, \\ USA; ${ }^{2}$ James Graham Brown Cancer \\ Center, University of Louisville, \\ Louisville, KY, USA; ${ }^{3}$ Department \\ of Pharmacology and Toxicology, \\ University of Louisville, Louisville, KY, \\ USA; ${ }^{4}$ Department of Microbiology \\ and Immunology, University of \\ Louisville, Louisville, KY, USA; ${ }^{5}$ Center \\ for Predictive Medicine, University of \\ Louisville, Louisville, KY, USA
}

\begin{abstract}
A variety of drug-delivery platforms have been employed to deliver therapeutic agents across cervicovaginal mucus (CVM) and the vaginal mucosa, offering the capability to increase the longevity and retention of active agents to treat infections of the female reproductive tract (FRT). Nanoparticles (NPs) have been shown to improve retention, diffusion, and cell-specific targeting via specific surface modifications, relative to other delivery platforms. In particular, polymeric NPs represent a promising option that has shown improved distribution through the CVM. These NPs are typically fabricated from nontoxic, non-inflammatory, US Food and Drug Administration-approved polymers that improve biocompatibility. This review summarizes recent experimental studies that have evaluated NP transport in the FRT, and highlights research areas that more thoroughly and efficiently inform polymeric NP design, including mathematical modeling. An overview of the in vitro, ex vivo, and in vivo NP studies conducted to date - whereby transport parameters are determined, extrapolated, and validated is presented first. The impact of different NP design features on transport through the FRT is summarized, and gaps that exist due to the limitations of iterative experimentation alone are identified. The potential of mathematical modeling to complement the characterization and evaluation of diffusion and transport of delivery vehicles and active agents through the CVM and mucosa is discussed. Lastly, potential advancements combining experimental and mathematical knowledge are suggested to inform next-generation NP designs, such that infections in the FRT may be more effectively treated.
\end{abstract}

Keywords: polymer nanoparticles, 3D cell culture, cervicovaginal mucus, CVM, transport, female reproductive tract, FRT, mathematical modeling, intravaginal delivery

\section{Introduction}

Mucosal tissue is a critical barrier that defends the body from pathogenic infection, and typically lines the surface of internal organs and body cavities. Within the female reproductive tract (FRT), the vaginal mucosa is composed of three layers that possess unique characteristics relevant to their protective functions (Figure 1). ${ }^{1,2}$ The secreted mucus layer, located on the apical side of the mucosa, is made of polymerized mucin fibers and globular secretions. Cervical mucus is produced by the ectocervix and forms cervicovaginal mucus (CVM) that locally lines the cervix, as well as the vaginal compartment. Beneath the mucus, within the vaginal compartment, lies the stratified epithelium, which is $\sim 200-400 \mu \mathrm{m}$ thick. The lamina propria (or stroma) lies at the basal portion of the mucosa and
Correspondence: jill M Steinbach-Rankins Department of Bioengineering, University of Louisville, 505 S Hancock, CTRB 623, Louisville, KY 40208, USA

$\mathrm{Tel}+\mathrm{I} 5028525486$

Fax + I 5028526806

Email jmstei0I@louisville.edu 


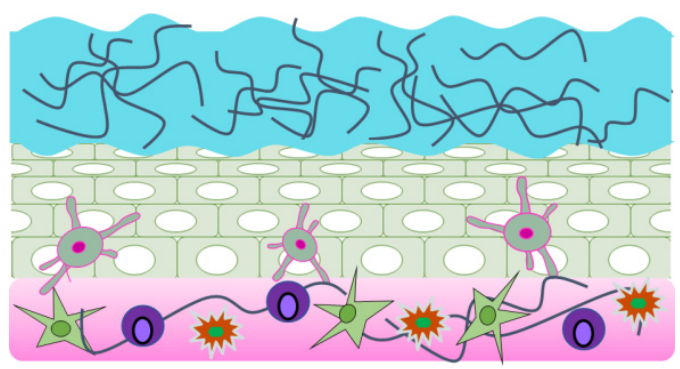

Figure I Schematic cross-section of the FRT. Mucus (top), epithelium (middle), and stromal layer containing immune cells and fibroblasts (bottom).

Abbreviation: FRT, female reproductive tract.

is the thickest $(2.5-3 \mathrm{~mm})$ and most complex of the three layers. Similar to the apical mucus, an array of mucin fibers can be found within the stroma. ${ }^{3}$ The arrangement of these mucin fibers - in parallel fiber networks or more randomized patterns - is influenced by menstrual and ovulation cycles. In addition, the stroma contains immune (eg, $\mathrm{CD}^{+}$and $\mathrm{CD}^{+} \mathrm{T}$ lymphocytes) and host (eg, macrophage, fibroblast) cells that are recruited to mediate the host-antimicrobial response.

The physiological and anatomical complexity of the FRT maintains the health of the microbiota and host cells by acting as a first line of defense against incoming pathogens. Yet, the effectiveness of the CVM as a barrier to infection can present significant challenges to the localized administration of microbicidal, antiviral, and antibiotic agents. Traditional dosage forms and delivery vehicles that deliver prophylactic and therapeutic active agents to the FRT such as intravaginal rings, gels, and films face numerous obstacles including: rapid clearance and dilution of topically applied active agents due to vaginal fluids, minimal diffusion through the CVM, poor transport to underlying tissue, and inadequate, heterogeneous agent distribution. To address these challenges, a variety of platforms have been developed to deliver therapeutic agents across the CVM, while also increasing the longevity and retention of active agents in the FRT. These platforms offer numerous advantages over traditional dosage forms by protecting active agents from degradation in the harsh acidic vaginal microenvironment, providing sustained release to improve the longevity and efficacy of the therapeutic payload, as well as enabling the high encapsulation and localized delivery of hydrophobic and hydrophilic agents (Table 1). For a more thorough review of microbicide delivery platforms, please refer to the studies. ${ }^{4-9}$

A promising platform that has been utilized to improve distribution through the CVM is based on polymeric nanoparticles (NPs). ${ }^{5-7,9-37}$ Due to small sizes, often ranging from 70 to $300 \mathrm{~nm}$, NPs exhibit unique physical properties that make them advantageous for delivery to the FRT, often providing desirable diffusion and transport kinetics through the CVM. By incorporating different surface modifications, NPs can improve retention, diffusion, and cell-specific targeting, relative to other delivery platforms. Additionally, polymeric NPs are typically made from nontoxic, non-inflammatory, US Food and Drug Administrationapproved polymers, which improve biocompatibility - a factor of significant importance due to the propensity for vaginal infections. The nanoscale properties, including high surface area to volume ratio, can impart favorable tunability in transport properties. This is achieved through NP surface modifications, by utilizing different surface chemistries to conjugate or adsorb ligands to the NP surface. These modifications can either enhance NP diffusion through the CVM via muco-inert properties, or can impart mucoadhesive characteristics for greater longevity in the basal mucus layer (Figure 2). ${ }^{5,18,19,23,31,32,36,38-40}$ Therefore, NPs can directly carry therapeutic payloads and navigate through the porous CVM, thereby increasing agent penetration and transport. Moreover, alterations in physicochemical properties can be adapted to attain desired pharmacokinetic (PK) and pharmacodynamic (PD) properties. $67,710,14,15,19,23,26$, 27,36,39,41 Thus, polymeric NPs can be designed to overcome transport barriers and to deliver effective doses to target sites, while maintaining agent activity and minimizing adverse effects on surrounding tissue.

To more effectively design delivery vehicles for application to the FRT, an in-depth understanding is needed of the anatomical and physiological structure of the vaginal microenvironment and how these structural characteristics may directly or indirectly affect NP diffusion and transport. A variety of studies have examined the impact of mucin fibers - found in the apical and basal layers of the mucosa on NP distribution and transport. ${ }^{12,42}$ Mucin, a major structural component of mucus, is a high-molecular-weight anionic molecule that non-covalently interacts to create a mesh-like framework. Depending on the arrangement, size, and porosity of these mucin fibers, active agent and delivery-vehicle penetration are impeded, similar to that of virus penetration. ${ }^{43-45}$ Additionally, the affinity for NPs to reversibly bind to mucin fibers has been studied and shown to impact NP diffusion. These studies, discussed in more detail later in the article, provide insight into the dependence of diffusion through the mucosa on ligand choice, surface modification density, and ligand molecular weight. Although NP 
Table I Overview of microbicide delivery vehicles currently used or investigated for delivery to the FRT

\begin{tabular}{|c|c|c|c|}
\hline $\begin{array}{l}\text { Microbicide delivery } \\
\text { platform }\end{array}$ & Attributes & Challenges & $\begin{array}{l}\text { Schematic } \\
\text { representation }\end{array}$ \\
\hline Intravaginal rings & $\begin{array}{l}\text { Lead in sustained-delivery, avoid leakage, coitus- } \\
\text { independent }\end{array}$ & $\begin{array}{l}\text { Incorporation of } \\
\text { biologics, alternative } \\
\text { delivery vehicle }\end{array}$ & \\
\hline Films & Convenient, portable, discreet, no leakage, low cost & Often transient release & \\
\hline Gels & $\begin{array}{l}\text { Convenient, easy to manufacture, mucosal adhesion } \\
\text { and spreading, comfort attributed to water content }\end{array}$ & $\begin{array}{l}\text { Often transient activity, } \\
\text { messiness due to } \\
\text { leakage }\end{array}$ & \\
\hline Nanoparticles & $\begin{array}{l}\text { Sustained-delivery, cell-specific targeting, incorporate } \\
\text { biologics, mobile platform for cell/tissue penetration } \\
\text { and uptake, potential as a coitus-independent platform }\end{array}$ & $\begin{array}{l}\text { Messiness and NP loss } \\
\text { due to leakage }\end{array}$ & \\
\hline Electrospun fibers & $\begin{array}{l}\text { Potential for sustained-delivery, alternative delivery } \\
\text { platform, potential as coitus-independent platform }\end{array}$ & $\begin{array}{l}\text { User preference and } \\
\text { comfort unknown }\end{array}$ & \\
\hline
\end{tabular}

Abbreviations: FRT, female reproductive tract; NP, nanoparticle.

diffusion and transport - as a function of these alterations have been experimentally studied in vitro and in vivo, limitless alterations exist, making it difficult to iteratively test all of the parameters that can impact binding, diffusion, and internalization.

In parallel with experimental design, mathematical modeling has been used to validate and predict factors that play pivotal roles in drug and NP distribution, particularly in the field of cancer. Mathematical models have been used to obtain more detailed insights into how specific parameters including size, shape, surface modifications, and drug loading can affect NP diffusion, without having to conduct exhaustive experiments to inform design. ${ }^{46-68}$

The goal of this review is to highlight recent experimental studies that have evaluated NP transport in the FRT. As there have been, to our knowledge, no mathematical studies evaluating NP transport in the FRT, we focus on modeling studies that have evaluated small-molecule transport and viral interactions, and describe research parameters and administration regimens that may be expanded upon to more thoroughly and efficiently inform potential NP design. We first present an overview of the in vitro, ex vivo, and in vivo polymeric NP studies conducted to date, by which transport parameters are determined, extrapolated, and validated. Second, we summarize how different NP design features have impacted FRT transport, and identify gaps that exist due to the limitations of iterative experimentation alone. We then discuss the potential of mathematical modeling as a complementary means to characterize and evaluate diffusion and transport of delivery vehicles and active agents through the CVM. Lastly, we suggest potential advancements combining mathematical and experimental knowledge to inform next-generation designs, such that optimal NP binding, diffusion, and internalization in the FRT may be achieved.

\section{Informing NP design with empirical studies \\ Introduction to factors that guide experimental studies}

As the field of intravaginal drug delivery has evolved, a variety of experimental methods have been established to study molecular and NP transport in vaginal mucosa models. In vitro, ex vivo, and in vivo methods have provided insight 
A

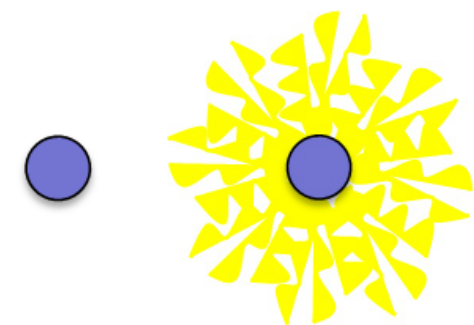

Unmodified Mucoadhesive

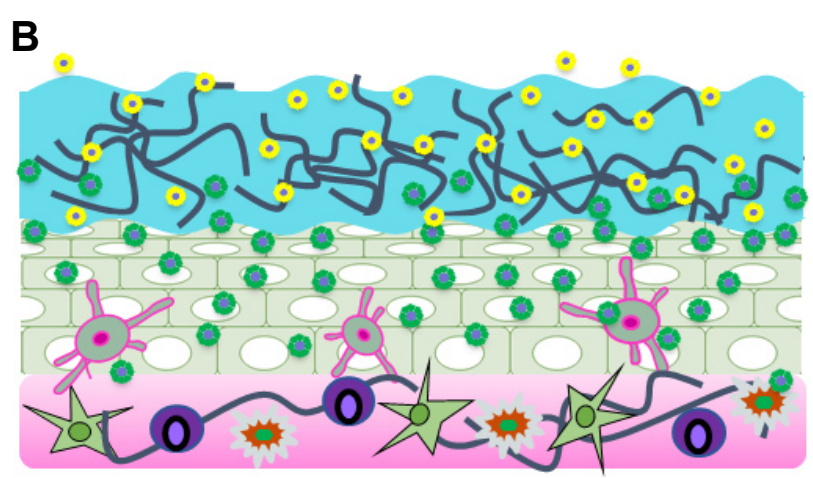

Figure 2 (A) Schematic of unmodified (blue only) and surface-modified mucoadhesive (yellow) and muco-inert (green) NPs. (B) Representative distribution of mucoadhesive and muco-inert NPs in the vaginal mucosa.

Abbreviation: NPs, nanoparticles.

into the pharmacologic behaviors of molecules and delivery vehicles, particularly their movement through the CVM. Each type of experimental model provides information on transport and the interactions that modulate this transport, with varying degrees of physiological complexity and accuracy.

The most controlled methods to study molecular diffusion and transport are provided by in vitro experimental models, wherein individual cell uptake and effect can be evaluated. However, monolayer cell culture often fails to adequately represent a system in which NPs undergo diffusive flux as they would in vivo, due to the inability of these systems to accurately represent three-dimensional (3D) physiologic architecture and microenvironmental conditions. To more faithfully represent the mucosal environment, more advanced in vitro systems such as synthetic mucus, ${ }^{69}$ undiluted CVM collected from human patients, ${ }^{11,20,30,31,35,70-72}$ and 3D cell culture techniques have been developed. . $7,28,52,55,73-77^{-10}$

In addition to utilizing a relevant model to study and obtain transport information, essential physical and chemical properties must be considered to effectively design NPs to navigate the CVM. Depending on whether mucoadhesive or muco-inert properties are preferred, these properties may be tailored to enhance distribution through mucus and to the underlying epithelial and stromal cell layers. A particular physical parameter - NP size - has been found to impact NP diffusion and transport kinetics, due to the pore size of the surrounding CVM. ${ }^{1,20,21,42}$ If NPs are larger than the average CVM pore diameter, effective transport may be hindered by physical and chemical interactions with mucin fibers. Contributing to these interactions, NP surface chemistry also impacts NP transport: surface charge, colloidal stability, surface-modifying agent of choice, molecular weight, and ligand surface density all affect NP interactions with the mucosa. ${ }^{11,18-20,24,25,30,31,34,35,37,40,43,72,78}$ These factors can influence how NPs, in a concentrationdependent manner, interact with one another.

Together, these factors contribute to the complexities associated with, first, understanding NP diffusion within the CVM and subsequently designing optimal NP delivery vehicles to meet the needs of a given application or physiological environment. Previous literature reviews $s^{5-7,9,15,17,25,32,38}$ have focused on the therapeutic efficacy of NP delivery systems and their potential applications in the FRT environment. Here, we highlight different in vitro, ex vivo, and in vivo techniques that have been implemented to better understand how the relevant parameters influence NP diffusion and transport kinetics in the CVM. In this section, we present these techniques and summarize how they have been utilized to gain deeper insights into the complexities of NP delivery within the FRT.

\section{In vitro transport studies}

\section{Human CVM studies}

Transport dependence on NP size and surface modification

Within the field of intravaginal drug delivery, CVM samples are often collected from patients and serve as important specimens to study NP transport and diffusion. This methodology provides a near-perfect physiological mucus model in which NP transport may be studied. 2,12,18,20,21,30,31,35,70,71 In a seminal study by Willits and Saltzman, fresh, mid-cycle cervical mucus was obtained from healthy human patients and used to investigate how mucus properties change on the basis of interactions with synthetic polymers. ${ }^{72}$ In the study, the addition of nonionic or cationic polymers such as polyethylene glycol (PEG) or polyvinylpyrrolidone (PVP) altered the gel-like structure of mucus. It was discovered that the addition of PEG $(3,350 \mathrm{Da})$ affected the structural properties of mucus by inducing hydrogen bond formation, leading to mucin fiber coalescence in regions that were in contact with the polymer and the resultant creation of larger pore sizes. In contrast, the addition of anionic polymers like poly(lactic-co-glycolic) acid (PLGA), had no effect on mucus properties. ${ }^{72}$ The inability of PLGA to induce structural changes within CVM may correlate with the observation that PLGA NPs tend to immobilize within the CVM, while conversely, the ability of PEG to alter mucin fiber arrangement may contribute to the enhanced diffusivity of 
PEG-modified NPs, as discussed in greater detail later in the article. The results from this pioneering study highlight the impact of polymer selection on NP-CVM interactions. ${ }^{72}$

In later work, human CVM was used in vitro to investigate the diffusion kinetics of different polymeric NP formulations, including polystyrene (PS) and PLGA NPs. ${ }^{20,21,29-31,34,35,37,79}$ In these studies, undiluted CVM secretions were collected from women who displayed healthy vaginal flora. Utilizing these specimens, Hanes et al investigated the relative diffusivities of different NP formulations..$^{20,21,29-31,34,35,37,79}$ Parameters that were of particular interest were NP size and the molecular weight and surface density of NP modifications, such as PEG. In one such study investigating the physical properties of NP size and surface charge, it was found that PS NPs (diameters spanning 200-500 nm) exhibited more rapid diffusion independent of surface chemistry and charge, relative to smaller, $100 \mathrm{~nm} \mathrm{NPs.} .^{20}$ This was contrary to the hypothesis that NPs with a diameter larger than the CVM pore size would have lower diffusion coefficients, relative to NPs with diameters smaller than the average pore size. PS NPs modified with PEG (PEGylated, $2 \mathrm{kDa}$ ) ranging in size from 100, 200, and $500 \mathrm{~nm}$ exhibited 20-, 400-, and 1,100-fold higher average mean squared displacements (MSDs) relative to unmodified carboxylated (PS-COOH) NPs of the same size, respectively. Furthermore, NPs with larger diameters and dense PEG coatings diffused through the CVM more rapidly, with diffusion coefficients reduced by 2,000-, 6-, and 4-fold for 100, 200, and $500 \mathrm{~nm}$ PEGylated NPs, respectively, relative to diffusion in water. In contrast, it was observed that PS-COOH NPs (without modification) were unable to diffuse through the CVM and were strongly mucoadhesive. ${ }^{20}$ This phenomenon was attributed to the addition of PEG, preventing polyvalent bond formation between PS NPs and hydrophobic mucin fiber domains. From this study, it was found that NP surface chemistry plays a vital role in diffusion through CVM.

To further explore the impact of NP size on CVM diffusion, a separate study investigated how the nanoscale mucus rheology undergoes changes upon exposure to differently sized non-mucoadhesive beads. In fresh, undiluted human CVM, the mucosa acted as an impermeable elastic barrier to non-mucoadhesive beads with diameters equal to or greater than $1 \mu \mathrm{m}$, whereas the CVM resembled a highly permeable viscoelastic liquid for beads with diameter less than or equal to $500 \mathrm{~nm}$. However, after the addition of a nonionic detergent, the CVM transitioned to an impermeable elastic barrier, even for the small beads. This change in mucus viscoelasticity is in accordance with the results from Lai et al, ${ }^{20}$ suggesting that NPs with diameters spanning 200-500 nm achieve maximum diffusion.
To gain further insight in regard to the role of mucin fiber pore size in NP diffusion and to expand on the study by Lai et al, ${ }^{20}$ experiments were conducted to investigate the effect of size on NP penetration through the CVM. ${ }^{21}$ PS NPs were modified with a high-density, low-molecular-weight, aminemodified PEG (2.0-3.4 kDa) to deter mucus adhesion. When observed in undiluted human CVM, PEG-modified PS NPs with sizes 100, 200, and $500 \mathrm{~nm}$ effectively penetrated mucus and exhibited diffusivities 6.5-, 3.5-, and 12-fold lower than that of NPs in water, respectively. However, PEG-modified PS NPs with a diameter of $1 \mu \mathrm{m}$ diffused 200-fold more slowly in mucus than in water. Using this information, the diffusion rates were fitted to a previously derived obstructionscaling model for cross-linked gels to determine the pore size of CVM. ${ }^{21,80}$ Although the pore size of undiluted human CVM was found to be $340 \pm 70 \mathrm{~nm}$, this wide distribution highlights the additional complexities of transport in the CVM. Additionally, the large difference in diffusion coefficients between the 0.5 and $1 \mu \mathrm{m}$ PS NPs emphasizes the importance of NP diameter in NP design.

In addition to NP size, ligand density and surface modification type play critical roles in the transport of NPs in human CVM. The effect of PEG as a surface modification for PLGA NPs and its impact on CVM binding and diffusion were investigated as a function of PEG molecular weight $(2,5$, and $10 \mathrm{kDa})$ and modification density $(5 \%-100 \%) .{ }^{12}$ As predicted by the investigators, the addition of PEG to the NP surface impacted the physical properties of the NPs by improving colloidal stability and neutralizing the NP surface charge. Moreover, PEGylated NPs were observed to have higher diffusion coefficients in human cervical mucus. Additionally, NPs partially modified with PEG (10\%) had a higher dependency on PEG molecular weight, whereas NPs partially modified (10\%) with 10-kDa PEG exhibited no difference relative to fully modified PEGylated NPs using 2- and 5-kDa PEG. ${ }^{12}$ Based on PEG molecular weight, partially modified PEGylated NP formulations (10\%) with 5- or 10-kDa PEG demonstrated statistically improved CVM diffusion relative to unmodified and 2-kDa partially modified PLGA NPs. These results highlight the interdependency of PEG molecular weight and modification density on NP diffusion through CVM.

\section{Transport dependence on NP formulation chemistry} As high PEG-modification density was observed to promote mucus penetration and decrease NP immobilization, studies sought to identify ways to maximize ligand conjugation to the NP surface. ${ }^{30,37}$ One of the challenges in obtaining high degrees of post-synthesis surface modification is that with 
conventional polymers, such as PS and PLGA, there are a limited number of surface groups available for conjugation, thereby limiting the density at which PEG (and other ligands) can be conjugated to the NP surface. To overcome this obstacle, PEG was covalently conjugated to the backbone of poly(sebacic acid) (PSA) to create a diblock copolymer that resulted in muco-inert NPs. ${ }^{30}$ The trajectories and diffusion coefficients of PSA-PEG, relative to unmodified PSA and PLGA NPs, were observed in undiluted human CVM. PSA-PEG NPs were significantly more mobile in CVM, having an MSD 400- and 230-fold higher than that of PSA and PLGA NPs, respectively, thereby confirming the correlation between high PEG density and rapid NP diffusion and transport through the CVM.

Another study investigated the use of biodegradable polymers to formulate diblock copolymers of PLGA $(18 \mathrm{kDa}$; lactic acid:glycolic acid $=50: 50)$ and $\mathrm{PEG}(2 \mathrm{kDa})$ to promote mucus-penetrating NP properties. ${ }^{37}$ The chemical structure of the diblock polymer - confirmed via proton nuclear magnetic resonance (H-NMR) and NP characterization with dynamic light scattering - revealed that PLGA and PLGA-PEG NPs possessed average hydrodynamic diameters of 130 and $90 \mathrm{~nm}$, respectively. To assess NP diffusion, fresh undiluted CVM was collected from human patients and, in this system, PLGA-PEG NPs were observed to diffuse much faster than PLGA NPs alone H-NMR with diffusion coefficients 8,000and 12,000-fold lower than in water, respectively. Based on these results, it was suggested that approximately $75 \%$ of PLGA-PEG NPs would penetrate a $10-\mu \mathrm{m}$ thick mucus layer in $30 \mathrm{~min}$, whereas PLGA NPs would exhibit negligible penetration within this duration. ${ }^{37}$ Due to the rapid clearance of the CVM - in the order of minutes to hours - it is highly desirable that topically administered NPs penetrate and diffuse into tissue prior to mucosal clearance. Furthermore, differences in the thickness of mucosal linings arising from different physiologic factors, such as age and stage of the menstrual cycle, vary from patient to patient. Therefore, these findings suggest that PEGylated NPs, created by presynthesis copolymerization, may avoid mucosal clearance whereas still providing adequate diffusion and penetration within the vaginal tissue. Both of these studies $^{30,37}$ reveal the impact that two different copolymer blends can have on formulating mucus-penetrating NPs while eliminating the need of post-synthesis surface modification.

\section{Transport dependence on NP formulation reactants}

The impact of residual reactants from NP synthesis was explored in relation to NP transport through the CVM. ${ }^{37,40,79}$
Surfactants that are typically used for NP synthesis, such as polyvinyl alcohol (PVA), are intrinsically mucoadhesive and, therefore, post-synthesis residue may impede NP transport through mucus. Given this, the effect of surfactants on CVM distribution was assessed by analyzing NP diffusion in human CVM specimens. PLGA NPs were synthesized with novel vitamin $\mathrm{E}$ surfactants that had been previously conjugated to a 1 - or 5-kDa methoxy-PEG-OH (PLGA/VP1K and VP5K, respectively) PVA surfactant alone, and $\mathrm{COOH}$-only modified (PS-COOH) or PEG-modified (2 kDa) PS NPs as controls. PLGA NPs synthesized using the VP1K surfactant exhibited no marked increase in CVM transport relative to unmodified $\mathrm{COOH}$-only PS NPs. ${ }^{40}$ However, increasing the PEG molecular weight to $5 \mathrm{kDa}$ significantly impacted diffusion. The MSD of PLGA/VP5K NPs in CVM was 210and 33-fold higher than that of PS-COOH and PLGA/VP1K NPs, respectively. Moreover, VP5K PLGA NPs were found to be the most resistant to mucoadhesion relative to VP1K and traditional PLGA NPs, confirming the importance of and sensitivity to surfactant and PEG molecular weight on CVM diffusion. ${ }^{40}$

Given the correlation between neutral surface modification and enhanced mucus penetration, other studies sought to investigate the effect of PVA alone on CVM transport. Due to the hydrophilic and neutral nature of PVA, it was hypothesized that coating PLGA and PS NPs with PVA may enhance NP diffusion. ${ }^{79}$ Interestingly, PS NPs coated with PVA - regardless of concentration $(0.01$ or $1 \% \mathrm{w} / \mathrm{v})$ or molecular weight $(2,6$, or $25 \mathrm{kDa})$ - remained immobilized in the CVM. When tested in fresh, undiluted human mucus samples, PS NPs that were synthesized with 2, 6, and 25-kDa PVA ( $1 \% \mathrm{w} / \mathrm{V}$ incubation concentration) had diffusion coefficients 23,000-, 14,000-, and 10,000-fold lower than their theoretical values in water, respectively. ${ }^{79}$ The effect of increasing PVA molecular weight decreased mucoadhesion and, therefore, increased diffusion. Whereas PLGA NPs formulated with PVA surfactants were immobilized in the CVM relative to the surfactant-free NPs and PEG-PLGA NPs, these results suggest that the inclusion of PVA as a surfactant or surface modification may promote mucoadhesivity that can be tailored with changes in molecular weight or concentration.

\section{Summary of human CVM studies}

The use of human CVM affords the possibility to emulate a physiologically relevant mucosal environment and to use this environment to gain an understanding of how physicochemical parameters impact NP diffusion in the CVM. 
While in vitro studies that utilize human CVM studies provide an informative system, there are drawbacks to this methodology. Sources of human CVM are not always readily available, and adequate amounts of CVM required to study multiple diffusion parameters can be challenging to obtain. Furthermore, patient samples are often inherently different from one another. Despite these drawbacks, utilizing human CVM is a relatively straightforward technique that allows for rapid data interpretation, which can be valuable to the design of NP formulations. With this technique, NP delivery vehicles can be precisely designed to obtain mucoadhesion (for retention) or mucus penetration (for distribution). By using human CVM, the reviewed studies were able to explore how NP size, formulation chemistry, formulation reactants, surface charge, surface-ligand density, ligand molecular weight, and surfactant may significantly impact NP diffusion in an environment closely corresponding to in vivo CVM conditions.

\section{Synthetic mucus studies}

Synthetic mucus formulations have been developed to study mucus rheology and the diffusion of small molecules and some delivery vehicles in vitro as a means to avoid the shortcomings of human CVM. ${ }^{15,16,70}$ Although these mucus formulations are not obtained from humans, they are able to recapitulate critical mucus properties such as microstructure and viscoelasticity. Synthetic mucus has the ability to adequately simulate bulk mucus properties, although it lacks critical components of a biologic system, such as innate physiological interactions and cellular-molecular components. Whereas the majority of studies have focused on the analysis of mucus microstructure, ${ }^{2,25,30,42,72}$ viral transport, ${ }^{9,22,43-45,71,81}$ or small-molecule diffusion, ${ }^{71,82,83}$ limited studies have used synthetic mucus to assess delivery vehicle transport.

\section{Transport dependence on NP formulation chemistry}

Most recently, the interactions of dapivirine-loaded polycaprolactone (PCL) NPs with mucin incorporated in simulated vaginal fluid were studied in a synthetic mucus environment. ${ }^{14-16}$ PCL NPs encapsulating dapivirine were evaluated due to their potential application as microbicides, and their demonstrated mucoadhesivity, sustained release, and retention within the CVM. To create synthetic mucus, mucin fibers were added to simulated vaginal fluid (SVF) $(1.5 \% \mathrm{w} / \mathrm{v})$ followed by bulk rheological analysis to confirm that the viscoelastic properties were comparable to human CVM. ${ }^{16}$ Using this in vitro model, it was observed that varying NP surface charge significantly contributes to altering the diffusion time across a $200 \mu \mathrm{L}$ layer of SVF in an eight-well imaging chamber. In this system, negatively charged NPs exhibited the most rapid diffusion. ${ }^{16}$

To expand on this work, the effect of NPs modified with cetyltrimethylammonium bromide (CTAB), poly(ethylene oxide) (PEO), and sodium lauryl sulfate (SLS) was assessed both in in vitro cell monolayers and ex vivo porcine vaginal mucosa - to increase dapivirine retention in the vaginal CVM. ${ }^{14}$ In cell monolayers composed of CaSki vaginal epithelial cells, PEO-PCL NPs exhibited the lowest apparent permeability coefficient of dapivirine $\left(2.2 \pm 0.2 \mathrm{~cm} / \mathrm{s} \times 10^{-6}\right)$ relative to free dapivirine $\left(3.0 \pm 0.3 \mathrm{~cm} / \mathrm{s} \times 10^{-6}\right)$, whereas CTAB NPs exhibited the highest dapivirine permeability coefficient $\left(4.6 \pm 0.5 \mathrm{~cm} / \mathrm{s} \times 10^{-6}\right)$. In subsequent studies, ex vivo vaginal porcine tissue was utilized to further explore how these surface modifications impacted dapivirine retention and NP diffusion. ${ }^{14}$ Permeability coefficients obtained ex vivo followed similar trends, with PEO-PCL NPs exhibiting a very low dapivirine permeability coefficient $\left(0.6 \pm 0.2 \mathrm{~cm} / \mathrm{s} \times 10^{-6}\right)$ and CTAB-PCL NPs diffusing more rapidly, with a dapivirine permeability coefficient of $3.0 \pm 0.3 \mathrm{~cm} / \mathrm{s} \times 10^{-6}$, relative to PEO-PCL NPs and free dapivirine. These results suggest that PCL NPs modified with PEO have the strongest mucoadhesion, whereas CTAB-PCL NPs diffuse more rapidly through the vaginal epithelium. ${ }^{14}$

Transport dependence on NP-modifying agent and surface charge

Similar to the results obtained with human CVM, it was observed that the NP-modifying agent and surface charge significantly contribute to NP affinity for mucus. ${ }^{14}$ Interestingly, PEO-modified NPs exhibited enhanced mucoadhesion properties in this synthetic CVM system, which is in contrast to the aforementioned studies wherein highmolecular-weight PEG (10-40 kDa) was utilized to decrease mucoadhesion. ${ }^{12,29,30}$ This discrepancy may be due to the difference between human and synthetic mucus, and highlights the complexity of NP-CVM interactions.

\section{Toward more realistic in vitro systems}

One of the main drawbacks of in vitro systems utilizing human or synthetic CVM is the challenge of accurately representing the in vivo environment - including the complex anatomical structure of the mucus and the underlying tissue that comprises the FRT. When delivered topically, it is often desirable for NPs to traverse mucus, withstand mucus shedding, and penetrate to the underlying epithelial and stromal tissue, depending on the intended cell or pathogen target. To complement analyses provided by human or synthetic 
CVM models, 3D models that include polymer matrices and multicellular layers - representative of epithelial and stromal layers - have been created to provide a noninvasive and physiologically relevant environment in which to study transport parameters. Although a comprehensive review of available cell culture models may be found in the studies by He et al, ${ }^{74}$ hallmark studies relevant to FRT delivery are included in several studies. .4, $69,73,74,76,77,84,85$

\section{Ex vivo transport studies}

In addition to human and synthetic mucus in vitro systems that have been employed to study NP transport, ex vivo models have been developed to gain insight into NP transport in the intravaginal environment. By integrating some of the attributes of in vitro and in vivo systems, ex vivo models can provide physiologically relevant tissue architectures, representative of the FRT microenvironment. In particular, porcine vaginal tissue has been used to investigate the mucoadhesive properties of NPs and other colloidal systems, as described in the studies by Pereira et $\mathrm{al}^{41}$ and van Eyk and van der Bijl. ${ }^{86}$

Similar methodologies have been used to investigate the efficacy of tenofovir-encapsulated chitosan NPs as a potential microbicide. ${ }^{87}$ Chitosan NPs were synthesized with varying diameters, with the smallest and largest NPs ranging from 188 to $900 \mathrm{~nm}$. Due to the inherent bioadhesive properties of chitosan, it was hypothesized that NP mucoadhesion would be size dependent. It was found that NPs with smaller diameters were twice as mucoadhesive relative to the larger NPs. ${ }^{87}$ These results are in agreement with previous work that found that PS NPs ranging from 200 to $500 \mathrm{~nm}$ exhibited more rapid diffusion relative to $100 \mathrm{~nm} \mathrm{NPs},{ }^{20}$ suggesting that physical properties such as size and NP material (here, PS or chitosan) can be used to tailor NP mucoadhesivity.

Although ex vivo systems provide a physiologically representative environment in which to study NP diffusion - having defined mucosal and underlying vaginal tissue layers - they still suffer from limitations, such as the inherent differences between animal and human tissues. Additionally, when studying diffusion in ex vivo models, there is an inability to temporally evaluate NP transport and clearance from the CVM, relative to vaginal fluid and tissue flux, because continual fluid exchange does not occur in these models.

\section{In vivo transport studies}

Although in vitro systems provide a highly controlled environment, spatiotemporal knowledge is seldom derived from in vitro cell monolayer studies due to the two-dimensional limitations and inherent differences of in vitro systems relative to in vivo tissue. Additionally, an in vitro system is typically limited to one or two cell types, or is focused on mucus alone, providing a comparatively isolated and compartmentalized method to investigate NP diffusion and transport. As such, in vivo systems can address the limitations of in vitro as well as ex vivo models, and enable the comprehensive assessment of how alterations in NP formulations impact transport in the CVM. A more comprehensive evaluation is possible due to the ability of in vivo models to provide a realistic environment in which to study NP diffusion and transport, and to evaluate the associated therapeutic efficacy within the FRT. In particular, tissue histology from in vivo studies provides knowledge of how drug-delivery vehicles diffuse and distribute throughout vaginal tissue. Such in vivo studies enable investigators to study the diffusion kinetics within the FRT as an integrated system, which provides a more thorough evaluation of how NP delivery systems interact with the CVM and vaginal tissue. With this in mind, the complexity of the in vivo environment requires the consideration of more parameters to rationally design NP delivery vehicles.

\section{Effects of surface-modification type and density on in vivo transport}

One way to improve intravaginal penetration and retention of polymeric NPs in vivo has been to conjugate NPs with ligands to modulate physicochemical properties, such as surface charge and hydrophobicity. To explore the effect of conjugating biotinylated PEG (2 $\mathrm{kDa})$ to avidin-modified PLGA NPs, a murine model was used to measure the vaginal retention of surface-modified NPs. ${ }^{10}$ NP groups, including avidin-only and avidin-PEG NPs, exhibited up to 5 times increased vaginal retention, relative to unmodified NPs. Furthermore, PEG NPs were found within the submucosal stromal cells and epithelium, suggesting that these NPs efficaciously penetrate the CVM to the underlying basal immune cells. Furthermore, relative to avidin-only and unmodified NPs, PEG NPs were found at higher concentrations throughout the CVM up to $6 \mathrm{~h}$ post-administration. ${ }^{10}$

Building upon this study, PLGA NPs with a variety of PEG modification densities (2, 3, 5, 8, 10, and $25 \mathrm{wt} \%$ ) were synthesized to study the impact of modification density on tissue penetration. ${ }^{34} \mathrm{NP}$ synthesis was accomplished by blending a diblock copolymer of $5 \mathrm{kDa}$ PEG and PLGA (5.6, 20, and $45 \mathrm{kDa})$, followed by confirmation of PEGmodification densities with H-NMR. The resulting NPs made from PEG-PLGA copolymers were tested in a murine model to assess NP diffusion and distribution within the CVM. 
Unmodified PLGA NPs were unable to fully penetrate vaginal rugae in the outermost epithelial layer, and remained sequestered in the outer mucus layer. Similarly, PEG surface densities below $10 \%$ were inefficient in penetrating the outer mucus layer. However, high surface-coating densities spanning 10\%-25\% effectively diffused through the outer mucus layer and distributed evenly throughout the rugae. Moreover, PEG-PLGA copolymer NPs with 25\% PEG surface density exhibited significantly more surface coverage than all other NP formulations. ${ }^{34}$

Comparing these results with previous in vitro work where it was observed that PLGANPs partially coated (10 wt $\%$ ) with $5 \mathrm{kDa}$ PEG achieved enhanced diffusion relative to unmodified PLGA NPs ${ }^{11,12}$ - a coating density of 10\%-25\% was needed to achieve improved CVM diffusion in this in vivo study. ${ }^{34}$ This difference suggests that the minimal surface coating density required for diffusion within the CVM and uniform coverage of vaginal tissue may be underestimated by in vitro studies.

To further characterize the impact of NP surface modifications on CVM transport in vivo, PS NPs were modified with various molecular weight PEG molecules, ranging from 5 to $40 \mathrm{kDa}$, as well as surface-modification densities (1.3-3; area covered by PEG/total surface area). ${ }^{24,29}$ NPs densely coated with $40 \mathrm{kDa}$ PEG exhibited the most effective transport and distribution through CVM (5-10 min post-administration). Densely modified NPs were shown to more uniformly cover the epithelium, independent of PEG molecular weight, suggesting that high-molecular-weight PEG can improve NP penetration and distribution through human CVM. These results confirm previous in vitro work, ${ }^{11,14,16,20,21,29-31,34,35,37,79}$ which showed that ligand molecular weight and surface density play key roles in tailoring the mucoadhesive/muco-inert properties of NPs.

In a separate in vivo study, the effect of coating PCL $(14.8 \mathrm{kDa})$ NPs with a triblock polymer of PEO and poly (propylene oxide) (PPO) (PEO-PPO-PEO), relative to the typically assessed low-molecular-weight PEG (2-10 kDa) was evaluated. ${ }^{13}$ In female Institute of Cancer Research mice, PEO-PCL NPs were localized in vaginal and uterine tissues $2 \mathrm{~h}$ post-administration, penetrating as much as $40 \mu \mathrm{m}$ through the vaginal tissue and distributing throughout the rugae. When comparing the diffusion coefficients of these PEO-PCL NPs (high-molecular-weight PEO) to previously studied PEG-modified PS NPs ( $2 \mathrm{kDa} P E G),{ }^{20}$ the PEO-PCL NPs were only 3-7 times slower, suggesting that - despite increased PEG molecular weight - the PEOmodified NPs adequately traversed the CVM and penetrated the vaginal epithelium.

\section{Evaluation of transport of unmodified NPs}

In parallel with in vitro, ex vivo, and in vivo studies that have demonstrated enhanced delivery of surface-modified relative to unmodified - NPs, recent work has shown that penetration and retention of polymeric NPs may still be attained with unmodified NPs. The transport and efficacy of unmodified siRNA PLGA NPs were assessed within the FRT by intravaginally administering sub-200 nm diameter fluorescently labeled NPs to female Institute of Cancer Research mice. ${ }^{33}$ NP distribution was assessed over 7 days, and the analysis of tissue cross-sections revealed regions of the vaginal tract in which NPs penetrated $75 \mu \mathrm{m}$ beneath the lumen. Furthermore, time-course studies using multiphoton microscopy revealed that NPs had penetrated up to $120 \mu \mathrm{m}$ below the luminal surface and were retained as long as 7 days post-treatment. ${ }^{33}$ These results suggest that unmodified NPs penetrated the CVM and distributed throughout the vaginal tract to deliver a sufficient therapeutic payload. Although NP penetration and retention were observed to induce therapeutic efficacy, the transport of surface-modified NP groups was not measured in comparison. The question whether surface modification improves penetration and efficacy relative to unmodified PLGA NPs requires further investigation.

\section{Effects of surfactant on in vivo transport}

Concurrent with the described work that has correlated surface modification to diffusion and distribution in the FRT, other studies have explored the effect of utilizing a biocompatible surfactant - pluronic F127 - to produce mucus-penetrating particles. ${ }^{36}$ To investigate the improved chemotherapeutic efficacy obtained from these particles, a commonly used chemotherapeutic - paclitaxel - was encapsulated in the F127-modified NPs. When administered intravaginally in TC-1 tumor-bearing mice, unmodified NPs aggregated within the vaginal lumen, preventing diffusion to the underlying epithelium, even after $20 \mathrm{~h}$. In contrast, NPs coated with F127 demonstrated significantly improved distribution and diffusion, uniformly dispersing through the CVM and penetrating to the underlying epithelial tissue. This enhanced diffusion translated to improved therapeutic efficacy, with F127-modified NPs reducing tumor growth by approximately $93 \%$ relative to the untreated control group, whereas unmodified NPs reduced the tumor load by only $42 \%$. Moreover, survival time was extended to 11 and 19 days - for unmodified and surface-modified NPs respectively - relative to a survival time of 9 days for the untreated mice. ${ }^{36}$ This study 
highlights the use of an unconventional surface modification to increase the chemotherapeutic efficacy of intravaginally administered drugs.

\section{Effects of mucus properties and administration solution on in vivo transport}

Many of these in vivo (and in vitro/ex vivo) studies have focused on surface ligands and surfactants to enhance NP distribution, but other methods have assessed the role of mucus properties to provide enhanced NP delivery to the FRT. Because both PEGylated PS and PLGA NPs have shown varying degrees of efficacy over a range of PEG molecular weights and surface densities, a murine model was used to study the conditions in which mucus-penetrating PEG NPs may be delivered most effectively. ${ }^{19}$ In this study, PS and PLGA NPs modified with 2 kDa PEG were administered intravaginally in hypotonic media, mimicking advective transport. To quantify the distribution of these NPs in vivo, vaginal and ectocervical tissues were excised and imaged via fluorescence microscopy. Both PEG-modified PS and PLGA NP groups - administered in hypotonic media - were shown to uniformly accumulate and coat the entire vaginal epithelium after only $10 \mathrm{~min}$. Furthermore, similarly administered surface-modified NPs penetrated more than $100 \mu \mathrm{m}$ into the CVM within $10 \mathrm{~min}$. As a function of surface modification, images revealed that $88 \%$ and $86 \%$ of the tissue surface were covered with PEG-modified NPs, whereas only $30 \%$ and $36 \%$ coverage was attained with unmodified NPs in vaginal and ectocervical tissues, respectively. ${ }^{19}$ These studies corroborate other in vitro and in vivo data to suggest the significant impact PEGylation has on penetration/distribution kinetics in CVM, and provide insight into the impact of administration methods and conditions on NP diffusion. Thus, exploiting the more rapid fluid influx of NPs into the vaginal epithelium that is observed using hypotonic conditions may provide an effective administration technique that relies on advective transport rather than diffusion alone.

Subsequent work studies by Ensign et al, ${ }^{88}$ building upon studies by Ensign et al, ${ }^{19}$ investigated the impact of fluid osmolality by utilizing modest to high hypotonic solutions to administer PEG-modified ( $5 \mathrm{kDa}$ ) PS NPs to the murine vaginal tract. ${ }^{88}$ When delivered in both high and low hypotonic solutions (20 and $220 \mathrm{mOsm} / \mathrm{kg}$, respectively), PEG NPs were shown to rapidly penetrate the CVM and vaginal lumen, covering $88 \%$ and $76 \%$ of the underlying vaginal epithelium. This was a significant increase relative to the isotonic solution (294 mOsm $/ \mathrm{kg}$ ), in which PEG NPs only covered $60 \%$ of the vaginal epithelium. In addition to enhanced distribution and penetration, PEG-modified NPs exhibited higher vaginal retention at $1 \mathrm{~h}$ post-treatment when delivered in a hypotonic solution. In non-ambulatory mice, $69 \%$ and $83 \%$ of NPs were retained for isotonic and hypotonic solutions, respectively, whereas for ambulatory mice, the retention rate for the isotonic solution was $22 \%$, and remained somewhat constant (75\%) for the hypotonic solution. In both cases, PEG NPs administered in a hypotonic solution were retained within the vaginal tract for longer durations, indicating that the rapid delivery of PEG NPs to the vaginal surface using a hypotonic solution promotes NP retention. ${ }^{88}$ These results confirm the enhanced distribution and penetration effects from using hypotonic solutions to administer NPs intravaginally. Moreover, they underscore that administration solution, in addition to NP surface modification, may have a significant impact on in vivo mucus properties, thereby impacting drug delivery and distribution through the CVM.

\section{Effects of polymer type on in vivo transport}

In addition to assessing surface modifications and delivery conditions, different polymeric delivery vehicles have been evaluated in murine models. ${ }^{23}$ A novel formulation, composed of phenylboronic NPs, was studied to improve NP mucoadhesion within the FRT. ${ }^{23}$ Due to the affinity of phenylboronic acid to form stable cyclic ester bonds with mucin, phenylboronic acid rich-NPs (PBNPs) were seen as an attractive option to formulate mucoadhesive delivery vehicles. Moreover, it was believed that modifying PBNPs with sodium sulfate would increase colloidal stability and form stable bonds with mucin fibers. In preliminary in vitro studies, PBNPs that were surface modified with sodium sulfate at 5 and $10 \mathrm{wt} \%$ (PBNP-S5 and -S10) exhibited increased mucin adsorption at vaginal $\mathrm{pH}$ relative to unmodified PBNPs. ${ }^{23}$ Following intravaginal administration in mice, both PBNPs and PBNP-S5 had increased vaginal retention up to $48 \mathrm{~h}$ post-treatment, whereas the amount of control solution significantly decreased between 0 and $6 \mathrm{~h}$ post-treatment. Although the in vitro and in vivo results show nuanced differences in mucoadhesion, these studies highlight the similar relationships that may govern NP distribution with different polymers. Moreover, these results confirm that differences observed within carefully controlled in vitro experiments may be less impactful in an in vivo environment where multiple factors govern distribution. 
Effects of the reproductive environment on in vivo transport

Although the focus of this review has been to convey design factors that can affect NP distribution and transport through the CVM in the FRT, it is also important to note that natural physiological changes in the CVM may affect NP diffusion. When translated to clinical application, a variety of differences exist in the human reproductive environment, contributing to increased variation of the FRT characteristics between patients. For example, age, hormone levels, and menstruation cycle are known to have a significant impact on CVM rheology and thickness, as well as the rate of vaginal fluid clearance. ${ }^{1,2,42}$ To explore the impact of menstrual cycle on NP distribution, an ex vivo experiment was conducted using CVM that had been excised from estrus-phase mice or from mice that had been pretreated with a progestin to mimic the diestrus phase of thicker CVM secretions. ${ }^{78}$ Using this model, the diffusivities of PS NPs surface modified with PEG (2 or $5 \mathrm{kDa}$ ) were assessed, highlighting the variation in NP diffusion as a function of estrous cycle. In estrus-phase vaginal tissue, 70\% of PEG-NPs exhibited higher effective diffusivities through the CVM relative to all NP formulations administered to diestrus-phase mice. Furthermore, PEG-NPs in diestrus-phase CVM had an MSD of at least 30-fold less than the same NPs in estrus-phase CVM. From the lower effective diffusivities of PEG-NPs in diestrus-phase CVM, it was deduced that NP movement is primarily due to thermal fluctuations of the mucus, and that NPs were likely trapped within the mucus mesh as a result of steric hindrance. ${ }^{78}$

\section{Summary of in vivo studies}

The reviewed in vivo studies highlight the complexities associated with the rational design of intravaginal NP delivery systems. Key polymeric NP parameters that have been evaluated in controlled in vitro conditions include surfacemodifying agent, surface-coating density, size, and surface charge. When evaluating NP design in the FRT in vivo, these parameters must also include variations between menstrual cycle phases, method of administration, vaginal fluid clearance and NP retention, homogeneous distribution throughout vaginal tissue, and penetration of NPs into the vaginal lumen and rugae. The information obtained from in vivo transport studies provides for a more detailed understanding of the impact that realistic and varied conditions present in the FRT may have on NP transport and efficacy through the CVM. Moreover, these studies enable more informed correlations to help to identify factors that differ between benchtop and translational experiments, and which may have significant effects on distribution and efficacy in the FRT.

\section{Mathematical modeling of small- molecule transport and antibody interactions within the FRT}

Mathematical modeling has been applied in the field of intravaginal drug delivery to gain insight into how FRT physiology impacts drug diffusion, transport, and dosage regimens. Mathematical models provide a means to efficiently test drug- and molecule-diffusion properties, simulating experimental conditions that might otherwise be challenging, labor-intensive, and inefficient to replicate at the bench. Nonetheless, to generate meaningful outputs from such models, the functional relationships must reflect the associated biology and the input parameters must be accurate. In particular, key input parameters such as diffusion and drug or delivery vehicle partition coefficients, which are necessary to model transport through the CVM, are typically derived and validated from experimental analysis.

\section{Application of mathematical modeling}

The modeling of drug diffusion within the FRT has historically focused on drug flow and distribution along the vaginal canal. ${ }^{1,3,42,52,89}$ Recently, mathematical modeling has been applied to assess molecule transport and viral interactions within the surrounding vaginal tissue and mucosa..$^{3,42,45,71,81,90}$ Even more recently, with the advent of drug-delivery vehicles and microbicides, the value of mathematical modeling for reproductive applications has been expanded. The development of microbicides for prophylactic and therapeutic approaches has seen significant growth in terms of developing drug-delivery platforms to improve intravaginal delivery against sexually transmitted infections. ${ }^{9,15}$ Some of these platforms, which are topically applied to the vagina - including intravaginal rings, films, gels and most recently fiber meshes - have the capacity to provide tunable release of therapeutic agents that diffuse through the vaginal epithelium to the underlying stroma. In the near future, it is expected that mathematical modeling will also provide insight into NP-mediated drug delivery through the CVM.

\section{Modeling of drug molecule diffusion}

Pioneering studies developed various mass transport equations that describe the PK and PD data of small drug molecules 
Table 2 Summary of main mathematical model equations

\begin{tabular}{|c|c|c|c|}
\hline Model & References & Main equations & $\begin{array}{l}\text { Model description } \\
\text { (computer program type) }\end{array}$ \\
\hline 1 & Katz et al ${ }^{1,3,82,90}$ & $\begin{array}{l}\frac{\partial C_{G}}{\partial t}=D_{G} \frac{\partial^{2} C_{G}}{\partial x^{2}}-k C_{G} \\
\frac{\partial C_{E}}{\partial t}=D_{E} \frac{\partial^{2} C_{E}}{\partial x^{2}} \\
\frac{\partial C_{S}}{\partial t}=D_{S} \frac{\partial^{2} C_{S}}{\partial x^{2}}-k_{B} C_{S}\end{array}$ & $\begin{array}{l}\text { Multi-compartmental model: } \\
\text { unsteady diffusion mass } \\
\text { transport for antiretroviral } \\
\text { through the CVM (MATLAB) }\end{array}$ \\
\hline 2 & Gao et $\mathrm{a}^{91}$ & $\begin{array}{l}\frac{\partial C_{G}}{\partial t}=\left.D_{E} \frac{4_{W}}{V_{G}} \int_{x=0}^{L} \frac{\partial C_{E}}{\partial y}\right|_{y=0} d x-k_{D} C_{G} \\
\frac{\partial C_{E}}{\partial t}=D_{E}\left(\frac{\partial^{2} C_{E}}{\partial x^{2}}+\frac{\partial^{2} C_{E}}{\partial y^{2}}\right)-k_{\text {on }}\left\{C_{E} \phi_{E}-\frac{C_{D P}}{r}\right\}+k_{\text {off }} C_{D P} \\
\frac{\partial C_{S}}{\partial t}=D_{S}\left(\frac{\partial^{2} C_{S}}{\partial x^{2}}+\frac{\partial^{2} C_{S}}{\partial y^{2}}\right)-k C_{S}-k_{o n}\left\{C_{S} \phi_{S}-\frac{C_{D P}}{r}\right\}+k_{\text {off }} C_{L} \\
V_{B} \frac{\partial C_{b}}{\partial t}=\int_{00}^{n d} \int_{B} k_{B} C_{S} d x d y-k_{L} C_{B} \\
\frac{\partial C_{D P}}{\partial t}=k_{o n}\left\{C_{T F V} \phi-\frac{C_{D P}}{r}\right\}-k_{\text {off }} C_{D P}\end{array}$ & $\begin{array}{l}\text { Multi-compartmental model: } \\
\text { unsteady convection- } \\
\text { diffusion mass transport for } \\
\text { antiretroviral through the } \\
\text { CVM (MATLAB) }\end{array}$ \\
\hline 3 & Wessler et $\mathrm{al}^{81}$ & $\begin{array}{l}\frac{\partial \vec{V}}{\partial t}=\mathbf{D} \frac{\partial^{2} \vec{V}}{\partial z^{2}}+\vec{f}(\vec{V}, u)-\vec{g}(\vec{V}, u) \\
\frac{\partial u}{\partial t}=D_{A b 0} \frac{\partial^{2} u}{\partial z^{2}}\end{array}$ & $\begin{array}{l}\text { Reaction-diffusion: } \\
\text { virus-antibody interactions in } \\
\text { the FRT (proprietary) }\end{array}$ \\
\hline 4 & Chen et $\mathrm{al}^{45}$ & $\frac{\partial \vec{V}}{\partial t}=\boldsymbol{D} \frac{\partial^{2} \vec{V}}{\partial z^{2}}-\vec{f}(\vec{V}(z, t))+\vec{g}(\vec{V}(z, t))$ & $\begin{array}{l}\text { Reaction-diffusion: virus- } \\
\text { antibody interactions in the } \\
\text { FRT (proprietary) }\end{array}$ \\
\hline
\end{tabular}

Note: Bold text signifies a vector.

Abbreviations: CVM, cervicovaginal mucus; FRT, female reproductive tract.

diffusing through the CVM after topical application (Table 2). Studies by Gao and Katz explored mathematical modeling as a tool to better elucidate the parameters governing intravaginal drug delivery. The group developed a multicompartmental model to study the PK and PD behavior and diffusion of the antiretroviral tenofovir in the CVM as it released from an intravaginal gel. ${ }^{3}$ A series of coupled, partial differential equations characterized the transport of tenofovir through each layer of the mucosa (Table 2, Model 1). The model incorporated equations to evaluate the subsequent uptake and clearance of tenofovir into the vascular and lymphatic systems after it penetrated through the mucosa. Initial studies conducted with this model focused on optimization to fit experimental PK data, and the predictive model outputs were found comparable to empirical data. From this analysis, the effect of differing biologic conditions between the cervicovaginal tissue layers, as well as the time required to achieve efficacious levels of therapeutic payload, was evaluated and confirmed when compared to concentrations measured from vaginal tissue biopsies. ${ }^{3}$
This multi-compartmental model was extended in the studies by Gao et al, ${ }^{91}$ to account for natural variations in the vaginal canal, and to include convective drug transport due to spreading of the application gel (Table 2, Model 2). A subsequent study conducted a global sensitivity analysis on this model using Sobol indices to further understand how variations in parameters, which describe the application gel and the physiologic environment, cause disturbances in the model output. ${ }^{90}$ From this analysis, it was observed that the model output was most sensitive to the parameters characterizing the initial drug concentration in the application gel, the partition coefficient of tenofovir in the epithelium, and the rate constant at which the gel was diluted due to vaginal fluid. Very recently, a model of PLGA polymer degradation ${ }^{92}$ was adapted to simulate degradation and release of TFV and tenofovir disoproxil fumarate (TDF) from an electrospun PLGA fiber. ${ }^{93}$ TFV and TDF are structurally similar molecules, yet with very different release and distribution profiles. The model output was then incorporated into a two-dimensional (2D) 
multi-compartmental diffusion model building upon the work by Gao and $\mathrm{Katz}^{3}$ to simulate the release of TFV from a fibrous mesh into the vaginal epithelium and stroma. This framework consisting of two coupled models offers the capability to study variation in fiber degradation and drug release, and how these parameters in turn may affect drug distribution in tissue. ${ }^{93}$

Studies conducted by Katz et al have highlighted the fundamental mass transport principles and applications related to the diffusion and convection of drugs and small molecules through the vaginal environment. ${ }^{1,82,89,91}$ This work has focused on the use of models to investigate the PK and PD of tenofovir - and its associated derivative, TDF - when topically delivered to the vagina using either a gel ${ }^{1,91}$ or intravaginal ring, ${ }^{1,89}$ with differing dosage regimens. The model showed that the use of intravaginal rings created an enhanced accumulation of activated TDF in the stromal compartment that was maintained for a prolonged duration of weeks to months. Conversely, gels achieved an equivalent concentration of active agent in the stroma in less than 1 day; however, it was necessary to apply the gel repeatedly to maintain this concentration for longer than $24 \mathrm{~h}$. This study demonstrated how a model can be utilized to characterize the transport behavior of small molecules and, furthermore, to identify efficacious delivery modalities for specific antiviral agents. ${ }^{1}$

\section{Modeling of virus-antibody interactions}

Mathematical models have also been used to study the transport of viruses through - and their interaction with biological molecules, such as antibodies, within - the CVM. Antibodies have recently emerged as a prophylactic approach to combat viral infection within the FRT. In conventional therapy, antibodies have been utilized to elicit a targeted immunogenic response ${ }^{81}$ However, antibodies can provide an additional means to prevent viral infection. Antibodies can reduce the diffusive flux of a given virus, such as the human immunodeficiency virus (HIV), by binding to receptors on the individual virions and to mucin fibers within the CVM. ${ }^{45,71,81}$ This coupled binding has provided a virus-trapping system, in which virus diffusion is hindered before reaching the vaginal stroma. ${ }^{81}$

Studies conducted by Wessler et al have complemented experimental work, by utilizing deterministic mathematical models to characterize virus immobilization by antibodies in CVM and to gain insight into the most effective parameters that reduce virus diffusion (Table 2, Model 3). ${ }^{81}$ The group developed a mathematical model to study the impact that certain parameters, such as the rate of mucin binding and affinity for virion binding, have on prophylaxis. Immunoglobulin $\mathrm{G}$ ( $\mathrm{IgG}$ ) was chosen due to its proven efficacy in saturating the CVM and hindering HIV diffusive flux. The model results indicate that, to maximize virus immobilization and minimize viral diffusive flux and thus infection, IgG antibodies must exhibit a high affinity for virion binding and a weak affinity for mucin binding.

In another study, this group applied a mathematical model to evaluate the potential effects of secretory immunoglobulin A (IgA) in the CVM as a means to agglutinate and prevent HIV transmission (Table 2, Model 4). ${ }^{45}$ The model investigated the mechanisms by which secretory IgA binds to HIV, causing agglutination and aggregation and, thus, preventing infection. Experimental data were used to simulate virion collision kinetics in a physiologically accurate environment. Outputs from the model suggest that, regardless of viral load, secretory IgA-related agglutination is most likely not the primary mechanism by which viral and pathogenic infection is mediated. Furthermore, the model implies that this agglutination phenomenon is most likely effective against pathogens where diffusion is characterized as other than Brownian motion or has a high propensity for collision and encounter rates. ${ }^{45}$

\section{Definition of key parameters}

Expanding modeling capabilities to more complex environments requires that predictive deterministic and mechanistic models be applied and adapted to fit empirical data, such that the PK and PD properties of small-molecule drug diffusion and transport may be better understood. Data gathered empirically in in vitro tissue mimics as well as in vivo or ex vivo tissue models are, therefore, critical to extrapolate key transport parameters. In particular, when building a model to predict drug delivery within and through the CVM, whether it be small-molecule release from a topically applied platform or free drug delivery, the associated parameters must be precisely defined because the model output is dependent upon and sensitive to these parameters. Further, the geometry and assumptions made about the environment when creating a model are critical to its effectiveness. In studies where small-molecule diffusion through mucus membranes has been modeled, 1,2,42,71,89 it was found that the orientation of the mucin fibers as well as mucus porosity play critical roles in defining models that yield accurate simulations. The vaginal mucosa, the physiological effects of menstruation on fluid volumes and pressures within the vaginal canal, the cell densities of the lamina 
propria and stromal layers, and the properties of the mucus gel itself can all significantly impact diffusive transport. These characteristics need to be accurately described by the model parameters and functional relationships. In addition to having an appropriate understanding of the environment being modeled, it is necessary to define the physicochemical and PK parameters of the therapeutic agent of interest. Further, in terms of delivery platforms, relevant parameters for fiber meshes include mesh thickness, fiber diameter, and geometry, whereas for NP-based transport, the characteristics of the delivery vehicle include lipophilicity, surface charge, molecular weight or average size, and diffusion coefficient. These parameters are typically measured and extrapolated from empirical data, as illustrated in the studies by Gao and $\mathrm{Katz}^{3}$ and Gao et al. ${ }^{91}$

\section{Summary of modeling of small-molecule transport and viral interactions within the FRT}

Although the mathematical modeling of small therapeutic molecules in the FRT - and, more specifically, through CVM - is in its nascent stages, substantial progress has been made to date. The modeling work has advanced the understanding of the characteristics of small-molecule diffusion as well as the associated PK and PD behaviors in the complex environment of the FRT. Accounting in more detail for the nonlinear characteristics of cervical mucus as well as natural variations in the vaginal canal may be necessary to obtain further insights. Modeling different cell types and their interactions, such as stromal and immune cells, may help to fine tune the therapeutic response. Tailoring model parameters for different drugs and therapy modalities would help to move this work toward predictive capability. In particular, more mobile delivery vehicles, such as polymeric NPs, could encapsulate and protect active agents from unwanted cellular interactions or degradation, and carry them through the mucosa. Mathematical modeling could be applied to tailor such NPs to have mucoadhesive properties, to "stick" to the mucus layer, slowly releasing drug at this location, or to utilize muco-inert features to penetrate the epithelium and release drug within the stroma. Further, the coupling of various models - such as drug release from electrospun fibers with drug diffusion within the FRT $^{93}$ - holds the promise for an integrated understanding of therapeutic efficacy. Ultimately, the development of modeling frameworks that include user, therapeutic, and physiological characteristics may offer practical tools to complement current clinical approaches by informing therapeutic design that is personalized to individual patient needs.

\section{Utilizing empirical studies and mathematical modeling as comple- mentary tools to inform NP design}

Topical drug delivery to the FRT is a burgeoning field with many applications spanning chemotherapeutic treatments to microbicidal interventions. Polymeric NPs have demonstrated promising potential for efficacious delivery to the FRT, by enhancing the diffusion of encapsulated agents through the CVM, in addition to imparting tailorable mucosal interactions. As has been addressed in this review, several experimental approaches - comprising in vitro, ex vivo, and in vivo systems - have been utilized to characterize NP delivery and transport through the vaginal mucosa. From these studies, it can be appreciated that NP-CVM interactions are complex, and that the parameters governing these interactions must be considered to rationally design efficacious NP delivery systems.

Several factors may enhance or impede NP diffusion in the CVM. The in vitro and ex vivo studies discussed herein highlight that NP diffusion through the mucosa is dependent on ligand choice, modification density, and molecular weight. Expanding upon these studies, in vivo experiments have provided more complex environments in which to assess NP transport and diffusion. In addition, in vivo studies have shown that NP transport varies as a function of the administration method (here, tonicity and advection) and menstrual cycle phase at the time of administration. Furthermore, these studies utilize a physiologically relevant environment to provide spatiotemporal details that relate NP diffusion to penetration depth and homogeneity of NP distribution within vaginal tissue.

Despite the relationships elucidated by in vitro, ex vivo, and in vivo experiments, one of the challenges facing experimental systems is the scarce number of specimens available. The limited number of samples - whether human or synthetic mucus, ex vivo tissue, or in vivo animal models - are unable to meet the experimental needs to test the vast number of possible parameter variations involved in NP design. Even if human specimens were plentiful, iterative experiments would be inefficient to evaluate single or multiple parameter variations across every parameter of interest. Furthermore, variations exist between human CVM samples for in vitro studies, ex vivo tissue, and in vivo animal tissue, which make it difficult to translate or scale-up for seamless comparison to the human FRT. Additionally, synthetic mucus and tissue samples derived from animals lack some of the complex physiological properties that are unique to the human FRT. 
Complementary to experimental models, mathematical modeling presents a platform that enables a systematic assessment of how different mechanisms and interactions affect diffusion and transport within the CVM. Mathematical models have been used to study the release characteristics of topically applied delivery systems (eg, intravaginal rings, gels) and small-molecule transport through the CVM (antibodies, antivirals). However, to our knowledge, there are no mathematical models that investigate the impact that the mucosal diffusion of NPs has on the delivery or efficacy of therapeutic encapsulants, nor those that distinguish NP diffusion from the PK of therapeutic payload release. Moreover, as the field expands to include more labile biological molecules (eg, oligonucleotides, peptides, proteins), physiological conditions will have an increased impact on molecule activity as a function of release and tissue/intracellular location. Similar to drug delivery, individual biologic agent characteristics will vary as a function of environment, but may more rapidly be affected on the basis of release, binding, and internalization. Furthermore, for next-generation multipurpose delivery platforms - where multiple active agents, multiple types of delivery vehicles, or both are combined - it will be beneficial to elucidate these interactions alone and in combination with predictive and validating design tools.

Although mathematical modeling faces difficult challenges, such as maintaining biological relevance and minimizing computational costs, it is the primary means through which complex system analysis can be conducted to gain further insight into in vitro, ex vivo, and in vivo data. Models can be built and tailored to integrate the knowledge acquired from experimental data and to enable the effective assessment of variation in system parameters. Furthermore, whereas current experimental techniques are often limited to evaluating one or, at most, two different parameters in one study, modeling provides a high-throughput means to systematically evaluate multiple parameters.

Given the complexity of the FRT environment, more comprehensive models may be required to accurately simulate vehicle parameters to effectively model NP transport. In particular, a model that integrates ligand density, molecular weight, and hydrodynamic NP size would be beneficial to account for variations in diffusion through the different layers and tissue types of the FRT. Furthermore, ligand (eg, molecular weight, charge, hydrophobicity, affinity) and polymer types used to fabricate delivery vehicles may be characterized and included in the model framework to highlight how these molecules interact with the mucosa. For instance, relationships between characteristics such as surface charge, hydro- or lipophilicity, propensity or rate of mucin binding, and self-aggregation may be of interest, as these have been observed to affect diffusive transport. Modeling these NP properties in relation to their mucosal interactions would result in a more comprehensive model and, thus, more translatable and insightful simulations.

As highlighted by some of the in vivo work summarized here, and in addition to the properties inherent to NP formulation and characterization, the method of NP administration also impacts transport. The in vivo studies have shown that the tonicity and osmolarity of the topically applied NP suspension have a significant impact on NP penetration and transport in the mucosa and vaginal tissue. More specifically, the tonicity and osmolarity dictate the predominant method of transport - usually, a combination of bulk fluid flow and diffusion - and, thus, the rate of NP transport and vaginal distribution. Despite this impact, these parameters have only been investigated in limited studies. Incorporating administration properties and methodology into predictive mathematical models may provide more insight into the extent of interdependency between NP properties and administration method.

In addition to NP-specific characteristics and delivery vehicle administration conditions, the biological and structural properties of the mucosa itself can affect NP diffusivity and are, thus, of significant interest to model. Alterations in mucin fiber arrangement and mucus viscosity are related to stage of menstrual cycle, age, and hormone levels - all of which vary from patient to patient. These patient-specific dependencies make it difficult to characterize their effects with the limited in vitro and in vivo experimental techniques and specimens currently available. Therefore, including these parameters in a mathematical model may enable a more thorough understanding of how anatomical and physiological properties impact the fate of NP delivery systems, which has yet to be fully characterized empirically. Additionally, future work may focus on addressing limitations such as emulating vaginal fluid clearance and the variations in mucus composition and tissue thickness, which prove difficult to recapitulate in vitro.

The current knowledge gaps or limited experimental data may be informed by mathematical modeling. As highlighted, many key parameters have been shown to impact transport in experimental systems. However, a limited combination of variables and conditions can be assessed experimentally. Mathematical modeling can guide this experimental effort by correlating relationships between parameters and identifying those that have the most impact on system properties. 
Moreover, comparisons across experimental model types (eg, ex vivo murine with in vivo murine with human clinical trials) may be made, and explored in greater depth via mathematical models that are used to collect, retain, and refine this information across studies. This integrative approach would more readily enable the rational design of tailored and personalized delivery systems that meet patient-specific needs. The evolution and connectivity of these models could have significant clinical impact, identifying new ways to target FRT tissue, and increase the efficacy of next-generation delivery systems.

In addition to NP characteristics, administration methods, and patient-specific features that broadly govern delivery, the studies conducted to date highlight select choices in NP design features. For example, currently, the majority of NP diffusion studies have investigated the use of muco-inert (PEG) or mucoadhesive (chitosan) surface modifications. However, within the field of NP delivery, other modifying agents have been utilized such as cell-penetrating peptides (CPPs), ${ }^{26-28}$ which have intracellular targets and promote rapid cellular internalization. As new biologic delivery needs will necessitate both transport and cell internalization, the balance between features imparted by multiple ligand types will be valuable to explore. To date, there are few studies that address how NP diffusion varies as a function of such ligand modification, with only cursory studies investigating overall surface charge. In addition to peptides that promote ubiquitous internalization, specific ligands that target epithelial or immune receptors, may be of interest to explore emerging therapies for reproductive cancers, to more effectively prevent virus uptake, or to provide virus-like targeting to host tissue. As such, next-generation delivery vehicles may rely on combinations of different ligands, with different molecular weights and characteristics than the typically modeled PEG. Moreover, tunable surface features may enable temporal changes to carriers (eg, pH-responsive ligand release or sheddable ligands) that change carrier transport as a function of location and time. As this type of drug delivery has applications in both cancer chemotherapy and microbicides, it would be advantageous to have an improved understanding of how these NP delivery systems transport and exert their effect within the FRT.

\section{Conclusion}

A variety of experimental approaches including in vitro, ex vivo, and in vivo systems have been utilized to better understand and characterize the complex interactions of polymeric NP delivery systems with the vaginal mucosa. Inherent limitations of these systems preclude a complete understanding of how NPs behave and interact within the human FRT. Mathematical modeling has emerged as a complementary tool to investigate transport in the FRT and expand the ability to understand the interdependent nature of parameters that arise from innate physiological, NP formulation, and administration regimen variations. Modeling work that focuses in greater detail on NP-specific properties such as ligand density, type, and hydrophobicity, in combination with polymeric material, offers the opportunity to streamline experimentation that investigates mucosal diffusion. In addition, incorporating the effect of active agent release and efficacy as a spatiotemporal function - while also integrating the more labile properties of promising new biologics - would lend insight to PK/PD properties. From a clinical perspective, an enhanced focus on different administration methods that provide improved efficacy and the integration of patient-specific physiological conditions would provide valuable information on how vehicle distribution and activity can be controlled. The complementary integration of experimentation with mathematical modeling may yet offer the most informed path to design NP-mediated therapeutic delivery for the effective and safe treatment of infections in the female reproductive tract.

\section{Acknowledgment}

We gratefully acknowledge the partial support of the work for JMSR and LBS by the Knights Templar Eye Foundation.

\section{Author contributions}

LBS researched the articles and drafted the manuscript. HBF participated in the manuscript design and coordination and drafted the manuscript. JMSR conceived the manuscript review, participated in its design and coordination, and drafted the manuscript. All authors contributed toward data analysis, drafting and critically revising the paper, gave final approval of the version to be published, and agree to be accountable for all aspects of the work.

\section{Disclosure}

The authors report no conflicts of interest in this work.

\section{References}

1. Katz DF, Yuan A, Gao Y. Vaginal drug distribution modeling. Adv Drug Deliv Rev. 2015;92:2-13.

2. Lai SK, Wang YY, Wirtz D, Hanes J. Micro- and macrorheology of mucus. Adv Drug Deliv Rev. 2009;61(2):86-100.

3. Gao Y, Katz DF. Multicompartmental pharmacokinetic model of tenofovir delivery by a vaginal gel. PLoS One. 2013;8(9):e74404.

4. Acartürk F. Mucoadhesive vaginal drug delivery systems. Recent Pat Drug Deliv Formul. 2009;3(3):193-205.

5. Caramella CM, Rossi S, Ferrari F, Bonferoni MC, Sandri G. Mucoadhesive and thermogelling systems for vaginal drug delivery. Adv Drug Deliv Rev. 2015;92:39-52. 
6. Ensign LM, Cone R, Hanes J. Nanoparticle-based drug delivery to the vagina: a review. J Control Release. 2014;190:500-514.

7. Mallipeddi R, Rohan LC. Nanoparticle-based vaginal drug delivery systems for HIV prevention. Expert Opin Drug Deliv. 2010;7(1):37-48.

8. Steinbach JM. Protein and oligonucleotide delivery systems for vaginal microbicides against viral STIs. Cell Mol Life Sci. 2015;72(3): 469-503.

9. Whaley KJ, Hanes J, Shattock R, Cone RA, Friend DR. Novel approaches to vaginal delivery and safety of microbicides: biopharmaceuticals, nanoparticles, and vaccines. Antiviral Res. 2010;88 (Suppl 1): S55-S66.

10. $\mathrm{Cu} \mathrm{Y,} \mathrm{Booth} \mathrm{CJ,} \mathrm{Saltzman} \mathrm{WM.} \mathrm{In} \mathrm{vivo} \mathrm{distribution} \mathrm{of} \mathrm{surface-}$ modified PLGA nanoparticles following intravaginal delivery. J Control Release. 2011;156(2):258-264.

11. $\mathrm{Cu}$ Y, Saltzman WM. Controlled surface modification with poly(ethylene)glycol enhances diffusion of PLGA nanoparticles in human cervical mucus. Mol Pharm. 2009;6(1):173-181.

12. $\mathrm{Cu}$ Y, Saltzman WM. Drug delivery: stealth particles give mucus the slip. Nat Mater. 2009;8(1):11-13.

13. das Neves J, Araújo F, Andrade F, Amiji M, Bahia MF, Sarmento B. Biodistribution and pharmacokinetics of dapivirine-loaded nanoparticles after vaginal delivery in mice. Pharm Res. 2014;31(7):1834-1845.

14. das Neves J, Araújo F, Andrade F, et al. In vitro and ex vivo evaluation of polymeric nanoparticles for vaginal and rectal delivery of the anti-HIV drug dapivirine. Mol Pharm. 2013;10(7):2793-2807.

15. das Neves J, Nunes R, Machado A, Sarmento B. Polymer-based nanocarriers for vaginal drug delivery. Adv Drug Deliv Rev. 2015;92:53-70.

16. das Neves J, Rocha CM, Gonçalves MP, et al. Interactions of microbicide nanoparticles with a simulated vaginal fluid. Mol Pharm. 2012; 9(11):3347-3356.

17. El-Hammadi MM, Arias JL. Nano-sized platforms for vaginal drug delivery. Curr Pharm Des. 2015;21(12):1633-1644.

18. Ensign LM, Lai SK, Wang YY, et al. Pretreatment of human cervicovaginal mucus with pluronic F127 enhances nanoparticle penetration without compromising mucus barrier properties to herpes simplex virus. Biomacromolecules. 2014;15(12):4403-4409.

19. Ensign LM, Tang BC, Wang YY, et al. Mucus-penetrating nanoparticles for vaginal drug delivery protect against herpes simplex virus. Sci Transl Med. 2012;4(138):138ra79.

20. Lai SK, O'Hanlon DE, Harrold S, et al. Rapid transport of large polymeric nanoparticles in fresh undiluted human mucus. Proc Natl Acad Sci U S A. 2007;104(5):1482-1487.

21. Lai SK, Wang YY, Hida K, Cone R, Hanes J. Nanoparticles reveal that human cervicovaginal mucus is riddled with pores larger than viruses. Proc Natl Acad Sci U S A. 2010;107(2):598-603.

22. Lembo D, Swaminathan S, Donalisio M, et al. Encapsulation of Acyclovir in new carboxylated cyclodextrin-based nanosponges improves the agent's antiviral efficacy. Int J Pharm. 2013;443(1-2):262-272.

23. Li C, Huang Z, Liu Z, et al. Sulfonate-modified phenylboronic acidrich nanoparticles as a novel mucoadhesive drug delivery system for vaginal administration of protein therapeutics: improved stability, mucin-dependent release and effective intravaginal placement. Int $J$ Nanomedicine. 2016;11:5917-5930. eCollection 2016.

24. Maisel K, Reddy M, Xu Q, et al. Nanoparticles coated with high molecular weight PEG penetrate mucus and provide uniform vaginal and colorectal distribution in vivo. Nanomedicine (Lond). 2016;11(11): 1337-1343.

25. Fröhlich E, Roblegg E. Mucus as barrier for drug delivery by nanoparticles. J Nanosci Nanotechnol. 2014;14(1):126-136.

26. Sims LB, Curtis LT, Frieboes HB, Steinbach-Rankins JM. Enhanced uptake and transport of PLGA-modified nanoparticles in cervical cancer. J Nanobiotechnol. 2016;14:33.

27. Sims LB, Huss MK, Frieboes HB, Steinbach-Rankins JM. Distribution of PLGA-modified nanoparticles in 3D cell culture models of hypovascularized tumor tissue. J Nanobiotechnol. 2017;15(1):67.

28. Steinbach JM, Seo YE, Saltzman WM. Cell penetrating peptidemodified poly(lactic-co-glycolic acid) nanoparticles with enhanced cell internalization. Acta Biomater. 2016;30:49-61.
29. Suk JS, Xu Q, Kim N, Hanes J, Ensign LM. PEGylation as a strategy for improving nanoparticle-based drug and gene delivery. Adv Drug Deliv Rev. 2016;99(Pt A):28-51.

30. Tang BC, Dawson M, Lai SK, et al. Biodegradable polymer nanoparticles that rapidly penetrate the human mucus barrier. Proc Natl Acad Sci U S A. 2009;106(46):19268-19273.

31. Wang YY, Lai SK, So C, Schneider C, Cone R, Hanes J. Mucoadhesive nanoparticles may disrupt the protective human mucus barrier by altering its microstructure. PLoS One. 2011;6(6):e21547.

32. Wong TW, Dhanawat M, Rathbone MJ. Vaginal drug delivery: strategies and concerns in polymeric nanoparticle development. Expert Opin Drug Deliv. 2014;11(9):1419-1434.

33. Woodrow KA, Cu Y, Booth CJ, Saucier-Sawyer JK, Wood MJ, Saltzman WM. Intravaginal gene silencing using biodegradable polymer nanoparticles densely loaded with small-interfering RNA. Nat Mater. 2009;8(6):526-533.

34. Xu Q, Ensign LM, Boylan NJ, et al. Impact of surface polyethylene glycol (PEG) density on biodegradable nanoparticle transport in mucus ex vivo and distribution in vivo. ACS Nano. 2015;9(9):9217-9227.

35. Yang M, Lai SK, Wang YY, et al. Biodegradable nanoparticles composed entirely of safe materials that rapidly penetrate human mucus. Angew Chem Int Ed Engl. 2011;50(11):2597-2600.

36. Yang M, Yu T, Wang YY, et al. Vaginal delivery of paclitaxel via nanoparticles with non-mucoadhesive surfaces suppresses cervical tumor growth. Adv Healthc Mater. 2014;3(7):1044-1052.

37. Yu T, Wang YY, Yang M, et al. Biodegradable mucus-penetrating nanoparticles composed of diblock copolymers of polyethylene glycol and poly(lactic-co-glycolic acid). Drug Deliv Transl Res. 2012;2(2):124-128.

38. das Neves J, Amiji M, Sarmento B. Mucoadhesive nanosystems for vaginal microbicide development: friend or foe? Wiley Interdiscip Rev Nanomed Nanobiotechnol. 2011;3(4):389-399.

39. Martin DT, Steinbach JM, Liu J, et al. Surface-modified nanoparticles enhance transurothelial penetration and delivery of survivin siRNA in treating bladder cancer. Mol Cancer Ther. 2014;13(1):71-81.

40. Mert O, Lai SK, Ensign L, et al. A poly(ethylene glycol)-based surfactant for formulation of drug-loaded mucus penetrating particles. J Control Release. 2012;157(3):455-460.

41. Pereira MN, Reis TA, Matos BN, Cunha-Filho M, Gratieri T, Gelfuso GM. Novel ex vivo protocol using porcine vagina to assess drug permeation from mucoadhesive and colloidal pharmaceutical systems. Colloids Surf B Biointerfaces. 2017;158:222-228.

42. $\mathrm{Cu}$ Y, Saltzman WM. Mathematical modeling of molecular diffusion through mucus. Adv Drug Deliv Rev. 2009;61(2):101-114.

43. Olmsted SS, Padgett JL, Yudin AI, Whaley KJ, Moench TR, Cone RA. Diffusion of macromolecules and virus-like particles in human cervical mucus. Biophys J. 2001;81(4):1930-1937.

44. Boukari H, Brichacek B, Stratton P, et al. Movements of HIV-virions in human cervical mucus. Biomacromolecules. 2009;10(9):2482-2488.

45. Chen A, McKinley SA, Shi F, et al. Modeling of virion collisions in cervicovaginal mucus reveals limits on agglutination as the protective mechanism of secretory immunoglobulin A. PLoS One. 2015;10(7):e131351.

46. Frieboes HB, Sinek JP, Nalcioglu O, Fruehauf JP, Cristini V. Nanotechnology in cancer drug therapy: a biocomputational approach. In: Ferrari M, Lee AP, Lee LJ, editors. BioMEMS and Biomedical Nanotechnology. Boston, MA: Springer; 2006.

47. Curtis LT, Rychahou P, Bae Y, Frieboes HB. A computational/ experimental assessment of antitumor activity of polymer nanoassemblies for $\mathrm{pH}$-controlled drug delivery to primary and metastatic tumors. Pharm Res. 2016;33(10):2552-2564.

48. Curtis LT, Wu M, Lowengrub J, Decuzzi P, Frieboes HB. Computational modeling of tumor response to drug release from vasculature-bound nanoparticles. PLoS One. 2015;10(12):e0144888.

49. Decuzzi P, Pasqualini R, Arap W, Ferrari M. Intravascular delivery of particulate systems: does geometry really matter? Pharm Res. 2009; 26(1):235-243.

50. England CG, Gobin AM, Frieboes HB. Evaluation of uptake and distribution of gold nanoparticles in solid tumors. Eur Phys J Plus. 2015; 130(11). pii: 231. 
51. Frieboes HB, Wu M, Lowengrub J, Decuzzi P, Cristini V. A computational model for predicting nanoparticle accumulation in tumor vasculature. PLoS One. 2013;8(2):e56876.

52. Gao Y, Li M, Chen B, et al. Predictive models of diffusive nanoparticle transport in 3-dimensional tumor cell spheroids. AAPS J. 2013;15(3): 816-831.

53. Leonard F, Curtis LT, Ware MJ, et al. Macrophage polarization contributes to the anti-tumoral efficacy of mesoporous nanovectors loaded with albumin-bound paclitaxel. Front Immunol. 2017;8:693.

54. Leonard F, Curtis LT, Yesantharao P, et al. Enhanced performance of macrophage-encapsulated nanoparticle albumin-bound-paclitaxel in hypo-perfused cancer lesions. Nanoscale. 2016;8(25):12544-12552.

55. Koren E, Torchilin VP. Cell-penetrating peptides: breaking through to the other side. Trends Mol Med.2012;18(7):385-393.

56. Li M, Reineke J. Mathematical modelling of nanoparticle biodistribution: extrapolation among intravenous, oral and pulmonary administration routes. Int J Nano Biomater. 2011;3(3):222-238.

57. Reichel D, Curtis LT, Ehlman E, et al. Development of halofluorochromic polymer nanoassemblies for the potential detection of liver metastatic colorectal cancer tumors using experimental and computational approaches. Pharm Res. 2017;34(11):2385-2402.

58. Sinek J, Frieboes H, Zheng X, Cristini V. Two-dimensional chemotherapy simulations demonstrate fundamental transport and tumor response limitations involving nanoparticles. Biomed Microdevices. 2004;6(4):297-309.

59. van de Ven AL, Abdollahi B, Martinez CJ, et al. Modeling of nanotherapeutics delivery based on tumor perfusion. New J Phys. 2013; 15:55004.

60. van de Ven AL, Wu M, Lowengrub J, et al. Integrated intravital microscopy and mathematical modeling to optimize nanotherapeutics delivery to tumors. AIP Adv. 2012;2(1):11208.

61. Wu M, Frieboes HB, Chaplain MA, McDougall SR, Cristini V, Lowengrub JS. The effect of interstitial pressure on therapeutic agent transport: coupling with the tumor blood and lymphatic vascular systems. J Theor Biol. 2014;355:194-207.

62. Curtis LT, England CG, Wu M, Lowengrub J, Frieboes HB. An interdisciplinary computational/experimental approach to evaluate drug-loaded gold nanoparticle tumor cytotoxicity. Nanomedicine (Lond). 2016; 11(3):197-216.

63. Curtis LT, Frieboes HB. The tumor microenvironment as a barrier to cancer nanotherapy. Adv Exp Med Biol. 2016;936:165-190.

64. Godin B, Driessen WH, Proneth B, et al. An integrated approach for the rational design of nanovectors for biomedical imaging and therapy. Adv Genet. 2010;69:31-64.

65. Kaddi CD, Phan JH, Wang MD. Computational nanomedicine: modeling of nanoparticle-mediated hyperthermal cancer therapy. Nanomedicine (Lond). 2013;8(8):1323-1333.

66. Li M, Czyszczon EA, Reineke JJ. Delineating intracellular pharmacokinetics of paclitaxel delivered by PLGA nanoparticles. Drug Deliv Transl Res. 2013;3(6):551-561.

67. Li M, Panagi Z, Avgoustakis K, Reineke J. Physiologically based pharmacokinetic modeling of PLGA nanoparticles with varied $\mathrm{mPEG}$ content. Int J Nanomedicine. 2012;7:1345-1356.

68. Lu M, Al-Jamal KT, Kostarelos K, Reineke J. Physiologically based pharmacokinetic modeling of nanoparticles. ACS Nano. 2010;4(11): 6303-6317.

69. Lorton SP, Kummerfeld HL, Foote RH. Polyacrylamide as a substitute for cervical mucus in sperm migration tests. Fertil Steril. 1981;35(2): $222-225$.

70. Lai SK, Wang YY, Cone R, Wirtz D, Hanes J. Altering mucus rheology to "solidify" human mucus at the nanoscale. PLoS One. 2009; 4(1):e4294.

71. Saltzman WM, Radomsky ML, Whaley KJ, Cone RA. Antibody diffusion in human cervical mucus. Biophys J. 1994;66(2 Pt 1):508-515.
72. Willits RK, Saltzman WM. Synthetic polymers alter the structure of cervical mucus. Biomaterials. 2001;22(5):445-452.

73. Gardner JK, Herbst-Kralovetz MM. Three-dimensional rotating wall vessel-derived cell culture models for studying virus-host interactions. Viruses. 2016;8(11). pii: E304.

74. He B, Chen G, Zeng Y. Three-dimensional cell culture models for investigating human viruses. Virol Sin. 2016;31(5):363-379.

75. Le TTD, Pham TH, Nguyen TN, Ngo THG, Hoang TMN, Le QH. Evaluation of anti-HER2 scFv-conjugated PLGA-PEG nanoparticles on 3D tumor spheroids of BT474 and HCT116 cancer cells. Adv Nat Sci Nanosci Nanotech. 2016;7(2):025004.

76. Sobel JD, Tchao R, Bozzola J, Levison ME, Kaye D. Adherence of bacteria to human vaginal epithelial-cell multilayer tissue-cultures. Clin Res. 1979;27(2):A481.

77. van Eyk AD, van der Bij1 P. The culture of human buccal and vaginal epithelial cells for permeability studies. SADJ. 1998;53(11): 497-503.

78. Ensign LM, Henning A, Schneider CS, et al. Ex vivo characterization of particle transport in mucus secretions coating freshly excised mucosal tissues. Mol Pharm. 2013;10(6):2176-2182.

79. Yang M, Lai SK, Yu T, et al. Nanoparticle penetration of human cervicovaginal mucus: the effect of polyvinyl alcohol. $J$ Control Release. 2014;192:202-208.

80. Amsden B. An obstruction-scaling model for diffusion in homogeneous hydrogels. Macromolecules. 1999;32(3):874-879.

81. Wessler T, Chen A, McKinley SA, Cone R, Forest G, Lai SK. Using computational modeling to optimize the design of antibodies that trap viruses in mucus. ACS Infect Dis. 2016;2(1):82-92.

82. Katz DF, Gao YJ, Chang S. Multi-compartmental transport of anti-HIV molecules into and through mucosa. Biophys J. 2012;102(3):594a.

83. Shen H, Hu Y, Saltzman WM. DNA diffusion in mucus: effect of size, topology of DNAs, and transfection reagents. Biophys J. 2006;91(2): 639-644.

84. Pyles RB, Vincent KL, Baum MM, et al. Cultivated vaginal microbiomes alter HIV-1 infection and antiretroviral efficacy in colonized epithelial multilayer cultures. PLoS One. 2014;9(3):e93419.

85. Sobel JD, Tchao R, Bozzola J, Levison ME, Kaye D. Human vaginal epithelial multilayer tissue-culture. In Vitro. 1979;15(12): 993-1000.

86. van Eyk $A D$, van der Bijl P. Porcine vaginal mucosa as an in vitro permeability model for human vaginal mucosa. Int J Pharm. 2005;305(1-2): 105-111.

87. Meng J, Sturgis TF, Youan BB. Engineering tenofovir loaded chitosan nanoparticles to maximize microbicide mucoadhesion. Eur J Pharm Sci. 2011;44(1-2):57-67.

88. Ensign LM, Hoen TE, Maisel K, Cone RA, Hanes JS. Enhanced vaginal drug delivery through the use of hypotonic formulations that induce fluid uptake. Biomaterials. 2013;34(28):6922-6929.

89. Geonnotti AR, Katz DF. Compartmental transport model of microbicide delivery by an intravaginal ring. J Pharm Sci. 2010;99(8): 3514-3521.

90. Jarrett AM, Gao Y, Hussaini MY, Cogan NG, Katz DF. Sensitivity analysis of a pharmacokinetic model of vaginal anti-HIV microbicide drug delivery. J Pharm Sci. 2016;105(5):1772-1778.

91. Gao Y, Yuan A, Chuchuen O, Ham A, Yang KH, Katz DF. Vaginal deployment and tenofovir delivery by microbicide gels. Drug Deliv Transl Res. 2015;5(3):279-294.

92. Casalini T, Rossi F, Lazzari S, Perale G, Masi M. Mathematical modeling of PLGA microparticles: from polymer degradation to drug release. Mol Pharm. 2014;11(11):4036-4048.

93. Halwes ME, Tyo KM, Steinbach-Rankins JM, Frieboes HB. Computational modeling of antiviral drug diffusion from poly(lactic-co-glycolicacid) fibers and multicompartment pharmacokinetics for application to the female reproductive tract. Mol Pharm. 2018;15(4):1534-1547. 
International Journal of Nanomedicine

Dovepress

\section{Publish your work in this journal}

The International Journal of Nanomedicine is an international, peerreviewed journal focusing on the application of nanotechnology in diagnostics, therapeutics, and drug delivery systems throughout the biomedical field. This journal is indexed on PubMed Central, MedLine, CAS, SciSearch ${ }^{\circledR}$, Current Contents ${ }^{\circledR} /$ Clinical Medicine,
Journal Citation Reports/Science Edition, EMBase, Scopus and the Elsevier Bibliographic databases. The manuscript management system is completely online and includes a very quick and fair peer-review system, which is all easy to use. Visit http://www.dovepress.com/ testimonials.php to read real quotes from published authors.

Submit your manuscript here: http://www.dovepress.com/international-journal-of-nanomedicine-journal 